\title{
Solid lipid nanoparticle suspension enhanced the therapeutic efficacy of praziquantel against tapeworm
}

This article was published in the following Dove Press journal:

International Journal of Nanomedicine

17 October 2011

Number of times this article has been viewed

\author{
Shuyu Xie ${ }^{1, *}$ \\ Baoliang Pan ${ }^{1, *}$ \\ Baoxin Shi \\ Zhuangzhi Zhang ${ }^{2}$ \\ Xu Zhang ${ }^{2}$ \\ Ming Wang' \\ Wenzhong Zhou' \\ 'Department of Preventive Veterinary \\ Medicine, College of Veterinary \\ Medicine, China Agricultural \\ University, Beijing, People's Republic \\ of China; ${ }^{2}$ Veterinary Research \\ Institute, Xinjiang Academy of Animal \\ Science, Xinjiang, People's Republic \\ of China \\ *These authors contributed equally \\ to this study
}

Correspondence: Wenzhong Zhou Department of Preventive Veterinary Medicine, College of Veterinary

Medicine, China Agricultural University,

2 Yuanmingyuan Road West,

Beijing I00193, People's

Republic of China

Tel +86 I0 62734702

Fax +86 I0 62734840

Email zhouwz@cau.edu.cn

Ming Wang

Department of Preventive Veterinary

Medicine, College of Veterinary

Medicine, China Agricultural University,

2 Yuanmingyuan Road West, Beijing

I00I 93, People's Republic of China

Email vetdean@cau.edu.cn
Abstract: Hydatid disease caused by tapeworm is an increasing public health and socioeconomic concern. In order to enhance the therapeutic efficacy of praziquantel (PZQ) against tapeworm, PZQ-loaded hydrogenated castor oil solid lipid nanoparticle (PZQ-HCO-SLN) suspension was prepared by a hot homogenization and ultrasonication method. The stability of the suspension at $4{ }^{\circ} \mathrm{C}$ and room temperature was evaluated by the physicochemical characteristics of the nanoparticles and in-vitro release pattern of the suspension. Pharmacokinetics was studied after subcutaneous administration of the suspension in dogs. The therapeutic effect of the novel formulation was evaluated in dogs naturally infected with Echinococcus granulosus. The results showed that the drug recovery of the suspension was $97.59 \% \pm 7.56 \%$. Nanoparticle diameter, polydispersivity index, and zeta potential were $263.00 \pm 11.15 \mathrm{~nm}, 0.34 \pm 0.06$, and $-11.57 \pm 1.12 \mathrm{mV}$, respectively and showed no significant changes after 4 months of storage at both $4^{\circ} \mathrm{C}$ and room temperature. The stored suspensions displayed similar in-vitro release patterns as that of the newly prepared one. SLNs increased the bioavailability of PZQ 5.67-fold and extended the mean residence time of the drug from 56.71 to 280.38 hours. Single subcutaneous administration of PZQ-HCO-SLN suspension obtained enhanced therapeutic efficacy against tapeworm in infected dogs. At the dose of $5 \mathrm{mg} / \mathrm{kg}$, the stool-ova reduction and negative conversion rates and tapeworm removal rate of the suspension were $100 \%$, while the native PZQ were $91.55 \%$, $87.5 \%$, and $66.7 \%$. When the dose reduced to $0.5 \mathrm{mg} / \mathrm{kg}$, the native drug showed no effect, but the suspension still got the same therapeutic efficacy as that of the $5 \mathrm{mg} / \mathrm{kg}$ native PZQ. These results demonstrate that the PZQ-HCO-SLN suspension is a promising formulation to enhance the therapeutic efficacy of PZQ.

Keywords: pharmacokinetics, hydatid disease, Echinococcus granulosus

\section{Introduction}

Hydatid disease is a cause of substantial morbidity and mortality in most of the world, including parts of Europe, North America, South America, Asia, and Africa. ${ }^{1-3}$ It is a chronic cyst-forming parasitic helminthic disease of human beings as well as domestic and wild ungulates. ${ }^{4}$ This disease can result in a $10 \%$ decrease in whole of life performance for infected animals (reduction in quality of meat, production of fiber, production of milk, and the number of surviving offspring). ${ }^{5}$ Dogs are the definitive hosts that harbor the adult stage of the parasite, thus prophylaxis and therapy of tapeworm infection in dogs is the decisive step avoiding transmission of this disease to humans and livestock. ${ }^{4,6}$ Many programs have been implemented to remove the parasite in different countries/areas, such as continuous dosing of praziquantel (PZQ ) tablets or baits to dogs once or twice a week for years, ${ }^{7,8}$ but control of the transmission of tapeworm 
infection is very difficult. For example, it took New Zealand and Tasmania more than 30 years to eliminate the parasite infection following dog-targeted chemotherapy control. ${ }^{8}$ The program in Uruguay had little effect on livestock or human cystic echinococcosis rates over the first 20 years of the campaign. ${ }^{9}$ Epidemiologically, human cystic echinococcosis occurs predominantly in poor agricultural and pastoral communities. ${ }^{7}$ The poverty often relates to the failure of the community to adopt control measures. Therefore, achieving a high level of disease transmission control, reducing the cost of a control program, and increasing compliance from dog owners should be considered.

PZQ is one of the first-line anthelmintic drugs for treating tapeworm infections and has become the cornerstone for hydatid control campaigns worldwide. ${ }^{10}$ It has potent cestocidal activity against Taenia taeniaeformis, Echinococcus granulosus, Mesocestoides vogae, M. corti, Dipylidium caninum, and Hymenolepis diminuta in animals and humans. ${ }^{11,12}$ PZQ is highly effective on mature and immature tapeworm species tested in mice, rats, cats, dogs, and sheep. ${ }^{11}$ Although PZQ is a very effective anthelmintic, it is practically insoluble in water and has poor bioavailability because of extensive hepatic first-pass metabolism and rapid clearance from the bloodstream with a terminal elimination half-life $\left(\mathrm{t}_{1 / 2(\mathrm{el})}\right)$ of 1-3 hours. ${ }^{13,14}$ Repeated administration of high dose PZQ over a long time for therapy of cestode infection is required. For example, the common usage of PZQ is up to $75 \mathrm{mg} / \mathrm{kg}$ per day for 15-20 days preoperatively for hydatid disease caused by Echinococcus spp. ${ }^{15}$ The dose of PZQ is $50 \mathrm{mg} / \mathrm{kg} /$ day for 15 days for the cysticercus stage of Taeniasolium, especially in its localization in the brain (neurocysticercosis). ${ }^{16}$ Frequent administration is not only time-wasting, but also costly. Moreover, repeated medication is too complex to efficiently achieve satisfactory mass control. The high daily dose might produce a slight transient disturbance in general wellbeing, such as tiredness, dizziness, nausea, and hangover feeling. ${ }^{17}$

To overcome these shortcomings, new strategies of delivery are necessary. Some researchers have developed the long-term sustained-release implantable PZQ-containing bar and PZQ-loaded sustained-release PCL implant. ${ }^{18,19}$ Liposomal formulations have also been prepared to improve the bioavailability, systemic circulation time, and cestocidal activity of PZQ. ${ }^{20,21}$

Previous studies demonstrate that solid lipid nanoparticles (SLNs) significantly prolong the systemic circulation time and increased the bioavailability of PZQ. ${ }^{22,23}$ The improved bioavailability and systemic circulation time of PZQ could enhance its therapeutic efficacy and reduce dose and administration frequency. SLNs are usually produced as either aqueous dispersions (suspensions) or dry powders. Suspensions are preferred with regard to the ease of handling (no reconstitution necessary) and for cost reasons (eg, cost of freeze drying). ${ }^{24}$ It has been reported that aqueous dispersions of SLNs were basically stable under the optimization of the storage conditions for up to 3 years; however, some systems show particle growth followed by gelation. ${ }^{24,25}$ Alternatively, the liquid can be converted into a dry product by spray drying or lyophilization to avoid occurring instabilities. ${ }^{24}$

In this work, a PZQ-loaded hydrogenated castor oil (HCO)-SLN (PZQ-HCO-SLN) suspension was prepared. The stability of the suspension was evaluated by physicochemical characteristics of the nanoparticles and in-vitro release property of the suspension. The pharmacokinetics and therapeutic efficacy of the suspension were studied in dogs.

\section{Materials and methods Materials}

$\mathrm{HCO}$ was purchased from Tongliao Tonghua Castor Chemical Co, Ltd (Inner Mongolia, China). PZQ reference standard was bought from China Institute of Veterinary Drug Control (Beijing, China). Native PZQ was obtained from Wuhan Kanglong Century Technology Development Co, Ltd (Wuhan, China). Poly vinyl alcohol (PVA) was purchased from Sigma (St Louis, MO). Methyl alcohol used for high performance liquid chromatography (HPLC) was of liquid chromatography grade, available from Tedia Company, Inc (Fairfield, OH). The water was prepared with a Millipore (Bedford, MA) Milli-Q ${ }^{\circledR}$ system.

\section{Preparation of PZQ-HCO-SLN suspension}

The PZQ-HCO-SLN suspension was prepared by the hot homogenization and ultrasonication method as described previously. ${ }^{23}$ Briefly, HCO (17 g) and PZQ (3 g) were added in a $250 \mathrm{~mL}$ beaker and put in a boiling water bath. After the lipid-drug mixture became a clear melting solution, $130 \mathrm{~mL}$ of boiling 1\% PVA solution was poured into the lipid phase under magnetic stirring, and sonicated for 20 minutes with a VCX 750 Vibra-Cell ${ }^{\mathrm{TM}}$ (Sonics and Materials, Inc, Newtown, $\mathrm{CT}$ ), using the $13 \mathrm{~mm}$ microprobe with amplitude $35 \%$, to form a nanoemulsion. The hot nanoemulsion was cooled down to obtain a nanoparticle suspension. The control nanoparticle suspension was prepared in the same way without adding $P Z Q$. 


\section{Scanning electron microscopy (SEM) of SLNs}

The morphology of nanoparticles was studied by SEM (SE S-3400N; Hitachi, Tokyo, Japan). Briefly, $2 \mu \mathrm{L}$ of the nanoparticle suspension was placed on a glass surface. After oven-drying, the samples were coated with gold using an ion sputter for 3 minutes and examined at an accelerating voltage of $20 \mathrm{kV}$.

\section{Determination of drug recovery}

To determine the drug recovery from the suspension, $0.2 \mathrm{~mL}$ suspension was added into a $15 \mathrm{~mL}$ tube containing $9.8 \mathrm{~mL}$ methyl alcohol. The tube was heated on a boiling water bath for 10 minutes and then centrifuged at 10,000 rpm (Centrifuge 5810 R; Eppendorf, Germany) for 25 minutes. The PZQ in the supernatant was measured by HPLC using a UV Detector at wavelength $215 \mathrm{~nm}$ (Shimadzu Corporation, Kyoto, Japan). The control nanoparticle suspension without PZQ was treated similarly and used as blank for the measurements. The assay was repeated three times using different samples from independent preparations. Drug recovery is defined as follows:

Drug recovery $=($ Weight of $P Z Q$ in suspension /Weight of PZQ added) $\times 100$.

\section{Determination of diameter, polydispersity index, and zeta potential of SLNs}

The diameter, polydispersity index, and zeta potential of nanoparticles was measured by photon correlation spectroscopy (PCS Zetasizer Nano ZS90; Malvern Instruments, Worcestershire, UK). The nanoparticle suspension was diluted by 60 times for the determination of particle size and polydispersity index, and diluted by 360 times for zeta potential determination to get optimum kilo counts per second of 20-400 for measurements.

\section{In-vitro release study}

PZQ-HCO-SLN suspension $(0.2 \mathrm{~mL})$ was added in $1.8 \mathrm{~mL}$ $0.9 \%(\mathrm{w} / \mathrm{v})$ sodium chloride $(\mathrm{NaCl})$ solution (donor solution) in a dialysis bag (molecular weight: 8000-14,400) and dialyzed against $45 \mathrm{~mL} 0.9 \%$ (w/v) $\mathrm{NaCl}$ solution (receiver solution) in a $50 \mathrm{~mL}$ tube at $37^{\circ} \mathrm{C}$ under magnetic stirring at $100 \mathrm{rpm}$. At fixed times, the receiver solution was taken for PZQ quantitation by HPLC, and the dialysis bag was transferred to another $50 \mathrm{~mL}$ tube containing $45 \mathrm{~mL}$ fresh solution. The control nanoparticle formulation without PZQ was treated similarly and used as blank for the measurements. The experiments were carried out in triplicate.

\section{Stability evaluation}

Stability of the nanoparticle suspension was evaluated after the samples were stored at $4^{\circ} \mathrm{C}$ and room temperature for 4 months. The nanoparticle diameter, polydispersity index, and zeta potential, and the in-vitro release patterns, were used for the evaluation of the physical stability of the nanoparticle suspension. The assay was repeated three times using different samples from independent preparations.

\section{Pharmacokinetic study}

Xinjiang indigenous dogs $(15-27 \mathrm{~kg})$ were fed at the Veterinary Research Institute of Animal Science Academy of Xinjiang Uygur Autonomous Region, China. All experimental protocols concerning the handling of dogs were in accordance with the requirements of the experimental animal ethics/Ethics Committee at Animal Science Academy of Xinjiang Uygur Autonomous Region. The animals were housed at room temperature with free access to a standard diet and water. Before initiation of the experiment, ten healthy dogs were randomly divided into two groups, with five animals in each group. A single dose $(5 \mathrm{mg} / \mathrm{kg}$ ) of PZQ-HCO-SLN suspension or native $\mathrm{PZQ}$ suspended in $0.9 \%$ sterile $\mathrm{NaCl}$ at drug concentration of $2 \%(\mathrm{w} / \mathrm{v})$ was then subcutaneously administered into the cervical area of the dogs. At different time points post injection, blood samples were taken from the vena cervicalis, and the drug levels in the plasma were assayed.

\section{HPLC assay}

PZQ concentrations in plasma were measured by HPLC. Sample extracts were prepared by mixing $1 \mathrm{~mL}$ plasma with $6 \mathrm{~mL}$ mixture of methyl tert-butyl ether and dichlormethane $(2: 1 \mathrm{v} / \mathrm{v})$. The mixtures were vortexed for 3 minutes to allow complete mixing, followed by centrifugation at 14,000 rpm (Sigma 1-14; Sartorius, Germany) for 25 minutes. The supernatant was then incubated in a fan-assisted oven $\left(65^{\circ} \mathrm{C}, 30\right.$ minutes $)$ and was dissolved in $300 \mu \mathrm{L}$ mobile phase. A $100 \mu \mathrm{L}$ aliquot was taken for HPLC analysis. Chromatographic conditions were as follows: reverse phase C18 column, VP-ODS, $250 \mathrm{~mm} \times 4.6 \mathrm{~mm}$ (Shimadzu Corporation, Kyoto, Japan); mobile phase, acetonitrile/Milli-Q water (60/40, v/v); flow rate, $1.0 \mathrm{~mL} / \mathrm{min}$; column temperature, $20^{\circ} \mathrm{C}$; and UV detector wavelength, $215 \mathrm{~nm}$. The plasma concentration of PZQ was found to be linear over the range 5-2000 ng/mL. The correlation coefficient was 0.9999. The quantification limit of the method for PZQ in plasma was $5 \mathrm{ng} / \mathrm{mL}$. The extraction recovery for the plasma 
PZQ from three concentrations (10, 100, and $500 \mathrm{ng} / \mathrm{mL})$ was $89.1 \% \pm 0.8 \%, 94.2 \% \pm 3.6 \%$, and $91.1 \% \pm 1.2 \%$, respectively. The relative standard deviations of accuracy and precision for three different plasma concentrations of PZQ (10, 100, and $500 \mathrm{ng} / \mathrm{mL}$ ) were $4.5 \%, 0.6 \%$, and $3.4 \%$, respectively, for inter-day analysis, and $1.9 \%, 3.8 \%$, and $1.3 \%$, respectively, for intra-day analysis.

\section{Pharmacokinetic analysis}

The plasma concentrations versus time data were analyzed based on noncompartmental pharmacokinetics using PK Solutions 2.0 (Ashland, $\mathrm{OH}$ ) software. The plasma drug concentrations over time data were used to calculate the area under the concentration-time curve $\left(\mathrm{AUC}_{0-\infty}\right)$ and the mean residence time (MRT) of the PZQ-HCO-SLN suspension and native $\mathrm{PZQ}$.

\section{Therapeutic trials}

The therapeutic trials of PZQ-HCO-SLN suspension were performed in Xinjiang indigenous adult dogs naturally infected with E. granulosus. Fecal samples were taken from each dog pretreatment to confirm the E. granulosus infection by epidemiology survey, egg microscopy, and coproantigen enzyme-linked immunosorbent assay determination. ${ }^{26,27}$ Forty infected dogs were randomized into five treatment groups, with eight animals per group. Two drug doses $(0.5 \mathrm{mg} / \mathrm{kg}$ and $5 \mathrm{mg} / \mathrm{kg}$ ) in the form of either PZQ-HCO-SLN suspension or native PZQ suspended in $0.9 \%$ sterile sodium chloride at drug concentration of $2 \%(\mathrm{w} / \mathrm{v})$ were subcutaneously administered into four groups of dogs. One untreated group was used as control. Fecal samples were taken daily from each animal for 1 week post-treatment. Samples were analyzed using the saturated sucrose flotation technique, ${ }^{28}$ and the number of eggs per gram of feces was calculated. At the end of the experiment, three dogs from each treatment were sacrificed and their intestines were examined for the presence of tapeworms. ${ }^{11}$ If no tapeworm was found, they were considered as removed by the drug. The efficacy of the treatment was determined as stool-ova reduction rate, ${ }^{29}$ stool-ova negative conversion rate, ${ }^{30}$ and tapeworm removal rate. ${ }^{11}$

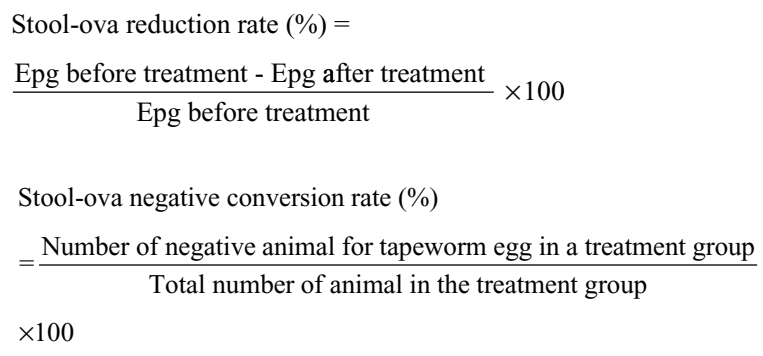

$$
\begin{aligned}
& \text { Tapeworm removal rate }(\%)= \\
& \frac{\text { Number of animal without E.granulosus in a treatment group }}{\text { Total number of animal in the treatment group }} \\
& \times 100
\end{aligned}
$$

where EPG is eggs per gram.

\section{Statistical methods}

The data on nanoparticle diameter, polydispersity index, zeta potential, and pharmacokinetic parameters of PZQ-HCOSLN suspension and native PZQ suspension were analyzed using one-way analysis of variance. Significance was evaluated at $P$-value of 0.05 . Statistical analysis was performed using Microsoft Excel (2003).

\section{Results \\ Physicochemical characteristics of the SLNs}

The drug recovery from the nanoparticle suspension was 97.59\%. SEM studies showed that the nanoparticles were spherical with smooth surfaces and had no differences after 4 months of storage at $4^{\circ} \mathrm{C}$ and room temperature (Figure 1). The diameter, polydispersivity index, and zeta potential of nanoparticles displayed no significant changes after 4 months of storage at $4{ }^{\circ} \mathrm{C}$ and room temperature as compared with that of the fresh preparation (Table 1).

\section{In-vitro release}

The stored nanoparticle suspension displayed the same release pattern as that of the fresh preparation. In-vitro release curve of the SLN suspension exhibited a biphasic pattern with a fast release from $55.33 \%$ to $58.20 \%$ within the initial 12 hours, followed by a slow and sustained release (Figure 2). The amount of cumulated release over 48 hours was $56.79 \%-60.69 \%$, and the daily release rate was lower than $0.5 \%$. In contrast, the release rate of native PZQ suspension was much faster. The cumulative release was up to $91.03 \%$ by 12 hours. The release almost completed (96.00\%) by 48 hours.

\section{Pharmacokinetics}

Plasma PZQ concentration-time curves are shown in (2) Figure 3. The pharmacokinetic parameters are depicted in Table 2. After subcutaneous administration of native PZQ, the plasma drug concentration reached a peak level of $47.82 \mathrm{ng} / \mathrm{mL}$ at 1.45 hours, then decreased to the quantification limit $(5 \mathrm{ng} / \mathrm{mL})$ by 48 hours and was undetectable by 72 hours. In the HCO-SLN suspension group, 



Figure I Scanning electron microscopy graphs of PZQ-HCO-SLN (65,000x): (A) Newly prepared SLNs, (B) SLNs stored at $4^{\circ} \mathrm{C}$ for 4 months; and (C) SLNs stored at room temperature for 4 months.

Abbreviations: PZQ-HCO-SLN, praziquantel-loaded hydrogenated castor oil solid lipid nanoparticle suspension; SLN, solid lipid nanoparticle.

PZQ reached a significantly higher peak concentration of $113.70 \mathrm{ng} / \mathrm{mL}$ at 1.93 hours and then decreased to the same level as that of native drug by 8 hours. The plasma drug concentration in the SLN group was higher than that of the native drug group again after 24 hours and maintained over $5 \mathrm{ng} / \mathrm{mL}$ for up to 264 hours. The plasma drug concentration was undetectable in the SLN group by 312 hours.

The pharmacokinetic analysis revealed that $t_{1 / 2(\text { el })}$ and MRT of PZQ-HCO-SLN were significantly longer than those of native PZQ (Table 2). Moreover, the $\mathrm{AUC}_{0-\infty}$ and maximal PZQ concentration $\left(\mathrm{C}_{\max }\right)$ of the PZQ-HCO-SLN suspension was 5.67- and 2.38-fold higher, respectively, than those obtained with the native PZQ (Table 2).

\section{Therapeutic efficacy}

The therapeutic efficacy in dogs naturally infected with E. granulosus is shown in Table 3. At PZQ dose of $5 \mathrm{mg} / \mathrm{kg}$, no egg was detected from the stool of dogs in the SLN group, while the stool-ova reduction and negative conversion rates in the native PZQ group were $91.55 \%$ and $87.5 \%$, respectively. When the drug dose reduced to $0.5 \mathrm{mg} / \mathrm{kg}$, the stool-ova reduction and negative conversion rates in the SLN group were $96.76 \%$ and $87.5 \%$, but they dropped dramatically to $22.08 \%$ and $0 \%$ in the native PZQ group.

Intestine examination showed that the tapeworm was only present in the stool-ova-positive dogs, therefore the

Table I The physicochemical characteristics of PZQ-HCO-SLN (mean $\pm S D, n=3$ )

\begin{tabular}{llll}
\hline Formulation & MD, $\mathbf{n m}$ & PDI & ZP, $\mathbf{m V}$ \\
\hline Newly prepared SLNs & $263.00 \pm 11.15$ & $0.34 \pm 0.06$ & $-11.57 \pm 1.12$ \\
SLNs stored at $4^{\circ} \mathrm{C}$ & $256.00 \pm 20.22$ & $0.37 \pm 0.05$ & $-10.87 \pm 0.7 \mathrm{I}$ \\
SLNs stored at room & $260.67 \pm 19.22$ & $0.36 \pm 0.09$ & $-12.33 \pm 0.8 \mathrm{I}$ \\
temperature & & & \\
\hline
\end{tabular}

Abbreviations: MD, mean diameter; PDI, polydispersity index; PZQ-HCO-SLN, praziquantel-loaded hydrogenated castor oil solid lipid nanoparticle suspension; SD, standard deviation; SLN, solid lipid nanoparticle; ZP, zeta potential. tapeworm removal rates with different treatment were the same as that of the stool-ova negative conversion rates in the sacrificed dogs.

\section{Discussion}

The physicochemical characteristics of the nanoparticle suspension were similar to that of the freeze-dried PZQ-HCOSLN prepared in the authors' previous study, and the drug recovery from the nanoparticle suspension was high. Due to the presence of unincorporated drug in the suspension, the initial release rate of the PZQ-HCO-SLN suspension within 2 hours was faster than that of lyophilized PZQ-HCO-SLN, but the subsequent release was very similar to that of freeze dried nanoparticles. ${ }^{23}$ The results demonstrated that the PZQ-HCO-SLN suspension is an effective sustained-release formulation.

The nanoparticle diameter, polydispersivity index, zeta potential, and release pattern have always been used to evaluate the stability of nanoparticle suspensions..$^{24,31}$ The HCO-SLN suspension did not have agglomeration,



Figure 2 In-vitro release of PZQ-HCO-SLN (mean $\pm S D, n=3$ ).

Abbreviations: PZQ, praziquantel; PZQ-HCO-SLN, praziquantel-loaded hydrogenated castor oil solid lipid nanoparticle suspension; SD, standard deviation; SLN, solid lipid nanoparticle. 

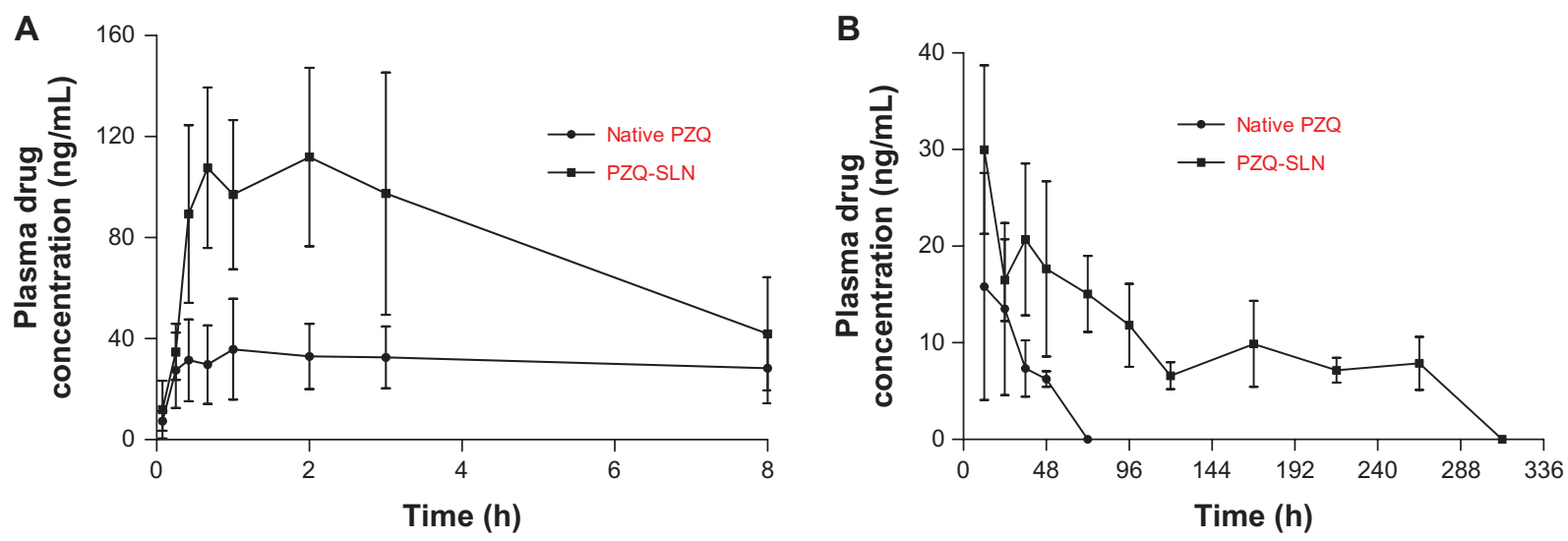

Figure 3 Plasma PZQ concentration-time curves after a single dose of subcutaneous administration of PZQ-HCO-SLN and PZQ suspension $(5 \mathrm{mg} / \mathrm{kg})(\mathrm{mean} \pm \mathrm{SD}, \mathrm{n}=5)$ : (A) within 8 hours, (B) from 12 to 312 hours.

Abbreviations: PZQ, praziquantel; SLN, solid lipid nanoparticle.

and the physicochemical characteristics had no significant changes after being stored at both $4^{\circ} \mathrm{C}$ and room temperature for 4 months, indicating the SLN suspension had good stability.

Subcutaneous administration of PZQ is one of the effective routes of cestode infection in animals. ${ }^{11,32}$ A previous study demonstrates that subcutaneous administration of PZQ-SLN is superior to oral and intramuscular routes for enhanced drug bioavailability and systemic circulation time. ${ }^{23}$ Therefore, the subcutaneous route was selected for pharmacokinetic study in this present work.

The PZQ-HCO-SLN suspension significantly extended the systemic circulation time and enhanced the bioavailability of PZQ. However, the $\mathrm{C}_{\max }$ of PZQ-HCO-SLN suspension was higher than that obtained with native PZQ, which is inconsistent with our previous study on lyophilized PZQ-HCO-SLN. This might be due to the free drug in the suspension, which could micronize or formulate pure drug nanoparticles in the preparation process, resulting in increased dissolution and swift absorption of the drug. In addition, the emulsifier could enhance the solubility of unentrapped drug, thus increasing the absorption rate of a drug at the injection site. ${ }^{33}$ Mass chemotherapy with PZQ can reduce the prevalence of tapeworm. ${ }^{34}$ However, the effect could only be sustained with continued regular use of PZQ baits. Once baiting ceases, animals become reinfected within a few months. ${ }^{34}$ The extended systemic circulation time of PZQ could decrease the dosage frequency, which is favorable to mass control of tapeworm infection.

E. granulosus prevalences are found in all continents and at least 100 countries. ${ }^{35}$ There are 22 provinces, autonomous regions, and municipalities in China reported with cystic echinococcosis, which is regarded as one of the major public health problems. ${ }^{36}$ In Xinjiang, the prevalence of cystic echinococcosis in humans, sheep, cattle, and dogs was $0.07-28.40,6.10-100.00,2.00-88.00$, and $0-51.80$, respectively. ${ }^{36}$ In this study, dogs infected with E. granulosus were chosen to evaluate the anthelmintic activity of the SLN suspension using two PZQ doses. The high dose of $5 \mathrm{mg} / \mathrm{kg}$ was based on the clinic dosage. ${ }^{11}$ Subcutaneous administration of $5 \mathrm{mg} / \mathrm{kg}$ is effective in eliminating tapeworm from dogs. ${ }^{37,38}$ Considering the enhancement of pharmacological activity of PZQ by SLNs, a low dose of $0.5 \mathrm{mg} / \mathrm{kg}$ was also used to assess the therapeutic efficacy of the SLN suspension. At both doses, the stool-ova reduction and negative conversion rates and tapeworm removal rate indicate that the SLN suspension could

Table 2 Pharmacokinetic parameters of PZQ-HCO-SLN and PZQ suspension after subcutaneous administration in dog $($ mean $\pm S D, n=5)$

\begin{tabular}{llllllll}
\hline Formulation & $\mathbf{t}_{1 / 2(\mathrm{ab})}$, hours & $\mathbf{t}_{1 / 2(\mathrm{~d})}$, hours & $\mathbf{t}_{1 /(\mathrm{el})}$, hours & $\mathbf{t}_{\max }$, hours & $\mathbf{C}_{\max }, \mathbf{n g} / \mathbf{m L}$ & $\mathbf{M R T}, \mathbf{h o u r s}$ & $\mathbf{A U C} \mathbf{C}_{0-\infty}, \mathbf{n g} \cdot \mathbf{h} / \mathbf{m L}$ \\
\hline PZQ-SLN & $0.31 \pm 0.11$ & $4.36 \pm 1.8 \mathrm{I}$ & $189.62 \pm 80.72^{\mathrm{a}}$ & $1.93 \pm 1.09$ & $113.70 \pm 37.27^{\mathrm{a}}$ & $280.38 \pm\left. 116.4\right|^{\mathrm{a}}$ & $5898.17 \pm 2048.73^{\mathrm{a}}$ \\
Native PZQ & $0.97 \pm 0.80$ & $2.60 \pm 0.79$ & $34.53 \pm 19.15$ & $1.45 \pm 1.07$ & $47.82 \pm 13.06$ & $56.7| \pm 23.4|$ & $1039.98 \pm 149.19$ \\
\hline
\end{tabular}



Abbreviations: $A \cup C_{0-\infty}$, area under the concentration-time curve from zero to infinity; $C_{\text {max }}$, maximal PZQ concentration in plasma; $M R T$, mean residence time; PZQ, praziquantel; PZQ-HCO-SLN, praziquantel-loaded hydrogenated castor oil solid lipid nanoparticle suspension; SD, standard deviation; SLN, solid lipid nanoparticle; $t_{1 / 2(a b)}$, absorption half-life; $t_{1 / 2(d)}$, distribution half life; $t_{1 / 2(e l)}$, elimination half-life; $t_{\max }$, time to reach $C_{\max }$. 
Table 3 Stool-ova reduction and negative conversion, and tapeworm removal rates post-treatment

\begin{tabular}{lllll}
\hline Treatment & $\begin{array}{l}\text { Dosage, } \\
\mathbf{m g} / \mathbf{k g}\end{array}$ & $\begin{array}{l}\text { Stool-ova reduction } \\
\text { rate, \% }\end{array}$ & $\begin{array}{l}\text { Stool-ova negative } \\
\text { conversion rate, \% }\end{array}$ & $\begin{array}{l}\text { Tapeworm } \\
\text { removal rate, \% }\end{array}$ \\
\hline PZQ-SLN & 5 & $100 \pm 0$ & $100(8 / 8)$ & $100(3 / 3)$ \\
PZQ-SLN & 0.5 & $96.76 \pm 9.16$ & $87.5(7 / 8)$ & $66.7(2 / 3)$ \\
Native PZQ & 5 & $91.55 \pm 23.88$ & $87.5(7 / 8)$ & $66.7(2 / 3)$ \\
Native PZQ & 0.5 & $22.08 \pm 29.42$ & $0(0 / 8)$ & $0(0 / 3)$ \\
Untreatment & 0 & $13.89 \pm 21.36$ & $0(0 / 8)$ & $0(0 / 3)$ \\
\hline
\end{tabular}

Abbreviations: PZQ, praziquantel; SLN, solid lipid nanoparticle.

significantly enhance the therapeutic efficacy and decrease the clinic dosage of PZQ. Since the cestocidal activity of PZQ is concentration- and time-dependent, ${ }^{11,21,28}$ exposure of the tapeworm to higher drug concentrations and/or longer time is essential for adequate treatment of parasite infection. In addition, the high drug concentration and long drug action time have a synergistic effect to enhance the cestocidal activity of PZQ. ${ }^{21}$ Based on the pharmacokinetics of the suspension and cestocidal mechanisms of PZQ, the enhanced cestocidal activity against other cestodes might be anticipated, but further studies need to be done to confirm its broad efficiency to treat tapeworm infections in most mammals.

\section{Conclusion}

The PZQ-HCO-SLN suspension has good stability stored at $4^{\circ} \mathrm{C}$ and room temperature. The SLN suspension significantly enhances the pharmacological activity and therapeutic efficacy of PZQ. The PZQ-HCO-SLN suspension would be a promising formulation for prophylaxis and therapy of tapeworm infection. The nanoparticle suspension would be an efficient alternative formulation for tapeworm transmission control.

\section{Acknowledgment}

This work was supported by the National Technology R\&D Program in the 11 th Five Year Plan of China (2006BAD04A16-41). The Earmarked fund for Modern Agro-insdustry Technology Research System. The Program for Cheung Scholar and Innovative Research Teams in Chinese Universities (No. IRT0866).

\section{Disclosure}

The authors declare no conflicts of interest.

\section{References}

1. Dakkak A. Echinococcosis/hydatidosis: a severe threat in Mediterranean countries. Vet Parasitol. 2010;174(1-2):2-11.

2. Anadol D, Özçelik U, Kiper N, Göçmen A. Treatment of hydatid disease. Paediatr Drugs. 2001;3(2):123-135.

3. Romig T, Dinkel A, Mackenstedt U. The present situation of echinococcosis in Europe. Parasitol Int. 2006;55 Suppl 1:S187-S191.
4. Jenkins DJ. Echinococcus granulosus in Australia, widespread and doing well! Parasitol Int. 2006;55 Suppl 1:S203-S206.

5. Battelli G. Evaluation of the economic costs of echinococcosis. Int Arch Hidatid. 1997;32:33-37.

6. Craig PS, Larrieu E. Control of cystic echinococcosis/hydatidosis: 1863-2002. Adv Parasitol. 2006;61:443-508.

7. Gemmell MA, Lawson JR, Roberts MG. Control of echinococcosis/ hydatidosis: present status of worldwide progress. Bull World Health Organ. 1986;64(3):333-339.

8. Craig PS, McManus DP, Lightowlers MW, et al. Prevention and control of cystic echinococcosis. Lancet Infect Dis. 2007;7(6):385-394.

9. Gemmell MA. Workshop summary: hydatid - new approaches. Vet Parasitol. 1994;54(1-3):295-296.

10. Urrea-París M, Casado N, Moreno M, Rodriguez-Caabeiro F. Chemoprophylactic praziquantel treatment in experimental hydatidosis. Parasitol Res. 2001;87(6):510-512.

11. Thomas H, Gönnert R. The efficacy of praziquantel against cestodes in animals. Z Parasitenk. 1977;52(2):117-127.

12. Gemmell MA, Johnstone PD, Oudemans G. The effect of praziquantel on Echinococcus granulosus, Taenia hydatigena and Taenia ovis infections in dogs. Res Vet Sci. 1977;23(1):121-123.

13. Caffrey CR. Chemotherapy of schistosomiasis: present and future. Curr Opin Chem Biol. 2007;11(4):433-439.

14. Cioli D, Pica-Mattoccia L. Praziquantel. Parasitol Res. 2003;90 Suppl 1: S3-S9.

15. Cobo F, Yarnoz C, Sesma B, et al. Albendazole plus praziquantel versus albendazole alone as a pre-operative treatment in intra-abdominal hydatisosis caused by Echinococcus granulosus. Trop Med Int Health. 1998;3:462-466.

16. Bale JF Jr. Cysticercosis. Curr Treat Options Neurol. 2000;2: 355-360.

17. Leopold G, Ungethüm W, Groll E, Diekmann HW, Nowak H, Wegner DHG. Clinical pharmacology in normal volunteers of praziquantel, a new drug against schistosomes and cestodes - an example of complex study covering both tolerance and pharmacokinetics. Eur J Clin Pharmacol. 1978;14:281-291.

18. Jiao W, Cheng F, Qun Q, et al. Epidemiological evaluations of the efficacy of slow-released praziquantel-medicated bars for dogs in the prevention and control of cystic echinococcosis in man and animals. Parasitol Int. 2005;54(4):231-236.

19. Cheng L, Lei L, Guo SR. In vitro and in vivo evaluation of praziquantel loaded implants based on PEG/PCL blends. Int J Pharm. 2010;387(1-2):129-138.

20. Mourao SC, Costa PI, Salgado HR, Gremiao MP. Improvement of antischistosomal activity of praziquantel by incorporation into phosphatidylcholine-containing liposomes. Int J Pharm. 2005;295(1-2): 157-162.

21. Hrcková G, Velebny S, Corba J. Effects of free and liposomized praziquantel on the surface morphology and motility of Mesocestoides vogae tetrathyridia (syn. M. corti; Cestoda: Cyclophyllidea) in vitro. Parasitol Res. 1998;84(3):230-238.

22. Yang L, Geng Y, Li H, Zhang Y, You J, Chang Y. Enhancement the oral bioavailability of praziquantel by incorporation into solid lipid nanoparticles. Pharmazie. 2009;64(2):86-89. 
23. Xie S, Pan B, Wang M, et al. Formulation, characterization and pharmacokinetics of praziquantel-loaded hydrogenated castor oil solid lipid nanoparticles. Nanomedicine. 2010;5(5):693-701.

24. Müller RH, Mader K, Gohla W. Solid lipid nanoparticles (SLN) for controlled drug delivery - a review of the state of the art. Eur J Pharm Biopharm. 2000;50(1):161-177.

25. Freitas C, Müller RH. Effect of light and temperature on zeta potential and physical stability in solid lipid nanoparticle (SLN $\left.{ }^{\mathrm{TM}}\right)$ dispersions. Int J Pharm. 1998;168(2):221-229.

26. Gulnur T, Mi XY, Zhang ZZ, et al. Using antibodies against tegument antigens for copro-ELISA to detect Echinococcus granulous in dogs. Acta Veterinaria et Zootechnica Sinica. 2011;42(6):845-850.

27. Zhang WB, Li J, McManus DP. Concepts in immunology and diagnosis of hydatid disease. Clin Microbiol Rev. 2003;16(1):18-36.

28. Dryden MW, Payn PA, Ridley R, Smith V. Comparison of common fecal flotation techniques for the recovery of parasite eggs and oocysts. Vet Ther. 2005;6(1):15-28.

29. Miró G, Mateo M, Montoya A, Vela E, Calonge R. Survey of intestinal parasites in stray dogs in the Madrid area and comparison of the efficacy of three anthelmintics in naturally infected dogs. Parasitol Res. 2007;100(2):317-320.

30. Slocombe JO, Heine J, Barutzki D, Slacek B. Clinical trials of efficacy of praziquantel horse paste $9 \%$ against tapeworms and its safety in horses. Vet Parasitol. 2007;144(3-4):366-370.
31. Dong Z, Xie SY, Zhu LY, Wang Y, Wang XF, Zhou WZ. Preparation and in vitro, in vivo evaluations of norfloxacin-loaded solid lipid nanopartices for oral delivery. Drug Deliv. 2011;18(6):441-450.

32. Papini R, Matteini A, Bandinelli P, Pampurini F, Mancianti F. Effectiveness of praziquantel for treatment of peritoneal larval cestodiasis in dogs: a case report. Vet Parasitol. 2010;170(1-2):158-161.

33. Müller RH, Radtke M, Wissing SA. Solid lipid nanoparticles (SLN) and nanostructured lipid carriers (NLC) in cosmetic and dermatological preparations. Adv Drug Deliver Rev. 2002;54 Suppl 1:S131-S155.

34. Schelling U, Frank W, Will R, Romig T, Lucius R. Chemotherapy with praziquantel has the potential to reduce the prevalence of Echinococcus multilocularis in wild foxes (Vulpes vulpes). Ann Trop Med Parasitol. 1997;91(2):179-186.

35. Eckert J, Deplazes P. Biological, epidemiological, and clinical aspects of echinococcosis, a zoonosis of increasing concern. Clin Microbiol Rev. 2004;17(1):107-135.

36. Wang ZH, Wang XM, Liu XQ. Echinococcosis in China, a review of the epidemiology of Echinococcus spp. Eco Health. 2008;5:115-126.

37. Andersen FL, Conder GA, Marsland WP. Efficacy of injectable and tablet formulations of praziquantel against mature Echinococcus granulosus. Amer J Vet Res. 1978;39(11):1861-1862.

38. Andersen FL, Conder GA, Marsland WP. Efficacy of injectable and tablet formulations of praziquantel against immature Echinococcus granulosus. Amer J Vet Res. 1979;40(5):700-701.
International Journal of Nanomedicine

\section{Publish your work in this journal}

The International Journal of Nanomedicine is an international, peerreviewed journal focusing on the application of nanotechnology in diagnostics, therapeutics, and drug delivery systems throughout the biomedical field. This journal is indexed on PubMed Central, MedLine, CAS, SciSearch $\AA$, Current Contents ${ }^{\circledR} /$ Clinical Medicine,

\section{Dovepress}

Journal Citation Reports/Science Edition, EMBase, Scopus and the Elsevier Bibliographic databases. The manuscript management system is completely online and includes a very quick and fair peer-review system, which is all easy to use. Visit http://www.dovepress.com/ testimonials.php to read real quotes from published authors. 